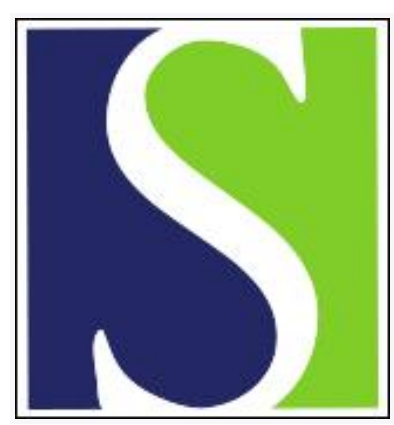

Scand J Work Environ Health 2016;42(6):561-562

https://doi.org/10.5271/sjweh.3596

Published online: 29 Sep 2016, Issue date: 01 Jun 2016

Reproductive health indicators and fetal medicine - many things will change

by Olsen J, Pedersen LH

Affiliation: Department of Clinical Epidemiology, Aarhus University, Olof Palmes Allé 43-45, 8200 Aarhus N, Denmark. jo@ph.au.dk

Key terms: fetal medicine; health indicator; perinatal epidemiology; reproductive epidemiology; reproductive health; reproductive health indicator; ultrasound

This article in PubMed: www.ncbi.nlm.nih.gov/pubmed/27685865

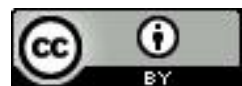




\section{Reproductive health indicators and fetal medicine - many things will change}

Reproductive epidemiologists study disease outcomes over three time periods: (i) from conception, or before, to birth, (ii) from birth to death and (iii) from death and into the next generations. They have traditionally been short of data from the time of conception to birth, and we use data at birth to estimate fetal growth or the incidence of congenital malformations. Although we are interested in incidence data for defects that start early in gestation, we have to use prevalence data at birth. Cumulative incidence will only be similar to prevalence at birth given no competing risks - or no fetal death after the onset of the lesion.

Routinely use of ultrasound methods in fetal medicine will change our monitoring of structural birth defects. We may now be able to link exposures to events with the right time sequence, for example on fetal growth deviations and get better data on fetal deaths also for twins and triplets. The scientific challenges will mainly come from induced abortions following ultrasound examinations.

Ultrasound data from the time of pregnancy will be of crucial importance for studies on fetal programming or "developmental origins of health and disease" (DOHaD). In humans, babies that are small at birth have an increased risk of, eg, cardiovascular disease, as shown by DJ Barker in the 1980s (1), but this association is probably not a direct consequence of the low birth weight but rather caused by external or internal exposures during fetal life. DOHaD studies that use outcomes at birth, including weight, as exposures or intermediates may be biased. One notorious example is the apparent protective effect of smoking on the mortality of children with a low birth weight (2). This bias, partly related to collider stratification bias, is potentially less important in studies using direct ultrasound assessments. The risk of reverse causation may also be reduced in longitudinal studies based on ultrasound data.

Fetal ultrasound examinations are also done to detect fetal structural abnormalities in order to start early treatment or terminate an effected pregnancy if that is permitted and requested by the parents. This change in timing and validity of determining congenital abnormalities (CA) will have substantial consequences for our monitoring of CA over time. Most of the existing monitoring systems are based on measuring prevalence of CA at time of birth, often allowing for a time period of detection from months to years since some of the CA are not detected at birth. They may be detected by ultrasound during gestation, but even for CA detectable in gestational weeks 20-24 and at birth, the sensitivity and specificity of times of diagnosing may differ so much that the measures are not comparable. Furthermore, the time from ultrasound to birth is sometimes interrupted by late fetal deaths and some of these deaths may be induced on indication. In any case, it will be difficult to reestablish long-term monitoring trends by applying birth correcting factors that will differ by the type of CA. We probably have to accept that long-term time trends need years to be reestablished and will have to be based on updated diagnostic facilities that will change over time. It may be difficult to spot increases in the incidence of CA in the future. An increase could be real or related to better diagnostic facilities operating in the time period from conception to birth.

Fetal medicine will sometimes make it possible to study causes and events in the proper time sequence, which is important since a cause has to precede an event as the only sine qua non causal criteria.

Measurements of recurrence "risks" of CA in families have always been complicated. It is well known that several CA have a tendency to be repeated in a subsequent pregnancy, most likely related to genetic factors or other time stable environmental exposures. Better diagnostic facilities with an option for an induced abortion may encourage high-risk parents to try to become pregnant and this may affect estimates of recurrence risk.

In any case, calculating recurrence risk for newborns following siblings with the $\mathrm{CA}$ in question will probably no longer work (maybe it never worked) since the desire to reach a given family size depends on many factors, including the perceived risk of a CA. Access to prenatal diagnostic data may therefore well produce data closer to recurrence risk than data recorded at the time of birth.

Pediatrics and Perinatal Epidemiology recently published a series of papers initiated by Olga Basso $(3,4)$ addressing in part the problem of moving from time scale one (starting at conception) to time scale two (starting at birth). Part of the addressed problems relate to a lack of options for starting observations on causal factors at the onset of exposure or, at best, before exposure. If that exposure happens early in fetal life, outcomes will be complicated by fetal deaths that probably end observation for $\geq 30 \%$ of subjects. That equals mortality rates we see for $\geq 95$-year-olds or equals a 
cumulative death risk seen for newborns from birth to $\geq 65$ years of age. If the exposure of interest is related to fetal death that opens up for strong collider stratification bias and selection when we condition on survival in our analyses for observations at the beginning of the second time scale (5). A negative association on that time scale need not reflect "prevention" in any sense other than suicides early in life will prevent later cancer deaths. It is difficult to imagine a counterfactual comparison to an exposed had he/she not been exposed and had survived fetal life. Those who were susceptible did not all survive. If we study fetal programming of adult diseases, we have to "condition on birth" in our studies, but we should be aware of the selection bias that follows.

Fetal medicine will in many ways produce better data or data we never have had before, but it will change the conditions in many aspects of reproductive epidemiology. The main advantage in analytical epidemiology is to get the time sequence right from exposure to outcome to avoid the problem of reverse causation and to do proper mediation analyses.

\section{Conflict of interest}

The authors declare no conflicts of interest.

\section{References}

1. Barker DJ, Gluckman PD, Godfrey KM, Harding JE, Owens JA, Robinson JS. Fetal nutrition and cardiovascular disease in adult life. Lancet 1993;341:938-41. http://dx.doi. org/10.1016/0140-6736(93)91224-A.

2. Wilcox AJ. On the importance--and the unimportance-of birthweight. Int J Epi. 2001;30:1233-41. http://dx.doi. org/10.1093/ije/30.6.1233.

3. Basso O. Implications of using a fetuses-at-risk aproach when fetuses are not at risk. Paediatr Perinat Epidemiol. 2016;30:310. http://dx.doi.org/10.1111/ppe.12254.

4. Basso O. No rates were harmed in making of this paper: Response to critiques. Paediatr Perinat Epidemiol. 2016;30:257. http://dx.doi.org/10.1111/ppe.12266.

5. Liew Z, Olsen J, Cui X, Ritz B, Arah OA. Bias from conditioning on live birth in pregnancy cohorts: an illustration based on neurodevelopment in children after prenatal exposure to organic pollutants. Int J Epidemiol. 2015;44:345-54. http:// dx.doi.org/10.1093/ije/dyu249.

Received for publication: 17 August 2016

Jørn Olsen, MD, PhD, ${ }^{1}$ Lars Henning Pedersen, MD, PhD ${ }^{2}$

1 Department of Clinical Epidemiology, Aarhus University, Aarhus N, Denmark.

2 Department of Clinical Medicine, Aarhus University / Department of Obstetrics and Gynecology, Aarhus University Hospital, Aarhus, Denmark.

Correspondence to: Dr Jørn Olsen, Department of Clinical Epidemiology, Aarhus University, Olof Palmes Allé 43-45, 8200 Aarhus N, Denmark. [Email: jo@ph.au.dk] 